Palimpsesto Vol. 10, № 17 (enero-junio, 2020): 39-49

Universidad de Santiago de Chile, ISSN 0718-5898

Marcela María Raggio

Universidad Nacional de Cuyo/CONICET

mraggio@mendoza-conicet.gob.ar

\title{
Redes interamericanas: la participación de Thomas Merton en Eco contemporáneo
}

\author{
Interamerican Networks: Thomas Merton's Contributions in Eco \\ Contemporáneo
}

\begin{abstract}
Resumen
Este artículo explora la revista argentina Eco Contemporáneo, publicación fundada y dirigida por Miguel Grinberg, con trece números aparecidos entre 1961 y 1969. El objetivo es detectar si en las contribuciones del estadounidense Thomas Merton, amigo y maestro de Grinberg, y en parte del corpus epistolar mertoniano, se advierten líneas directamente compartidas con el programa ideológico y cultural de Eco. De esta manera, se contribuirá al estudio de las redes conformadas por estos dos actores culturales de los años 60', que en conjunto con otros escritores e intelectuales norte y sudamericanos llevaron a cabo encuentros, publicaciones y proyectos que modularon el imaginario americano de la década en cuestión desde lo literario, cultural, político e ideológico.
\end{abstract}

Palabras claves: Eco Contemporáneo, Thomas Merton, Miguel Grinberg.

\begin{abstract}
This paper explores Eco Contemporáneo, an Argentine journal founded and directed by Miguel Grinberg, with thirteen issues published between 1961 and 1969. The main objective is to detect lines of continuity between Thomas Merton's letters and contributions to the journal and Eco's ideological and cultural program. In this way, we aim to contribute to the study of networks formed by these two cultural actors of the 1960s who, together with other writers and intellectuals from North and South America conducted gatherings, publications and projects that would define the decade's American image from a literary, cultural, ideological and political perspective.
\end{abstract}

Keywords: Eco Contemporáneo, Thomas Merton, Miguel Grinberg. 


\section{Introducción}

Entre 1961 y 1969 apareció la revista Eco Contemporáneo, editada desde Buenos Aires por Miguel Grinberg. Ezequiel Gatto sostiene que la revista es una de las principales prácticas contraculturales en Argentina en los años 60 (2012, p.173). De los diversos aspectos que pueden considerarse en el abordaje de esta revista, nos interesan las redes que se constituyeron en torno de ella, particularmente a partir del vínculo entre el director, Grinberg, y el norteamericano Thomas Merton (1915-1968).

Gatto define esas redes a partir de la noción acuñada por el propio Grinberg y sus contemporáneos:

[...] la revista fomentó y se inscribió en una suerte de fraternidad difusa que problematizó las relaciones entre creación y emancipación social. Dicha fraternidad [...] excedía las fronteras nacionales, haciendo de Eco Contemporáneo una presencia relacionada con individuos, grupos y colectivos distribuidos por el mundo. (2012, p.174)

La fraternidad, que sería llamada formalmente Movimiento Nueva Solidaridad, rechazaba enfáticamente formas organizativas que pudieran derivar en órganos burocráticos, y a la vez proponía relaciones inter-americanas, no solamente latinoamericanas. (Manzano, pp.116-117) Este punto nos interesa fundamentalmente, ya que más allá de la conexión con los poetas beats que ha señalado la crítica (Gatto 2010 y 2012; Manzano 2017), el monje, escritor místico, poeta y actor social Thomas Merton fue a la vez una especie de padrino del movimiento, guía y maestro, interlocutor de Grinberg y colaborador de Eco Contemporáneo.

Revisar los archivos permite determinar diferentes momentos (no necesariamente consecutivos cronológicamente) en la relación entre Miguel Grinberg y Thomas Merton. Originalmente, el argentino contacta a Merton, y llega a visitarlo en Gethsemaní, la abadía de Kentucky donde residía el monje. Luego de esa visita, el intercambio epistolar pone de relieve los emprendimientos literarios contraculturales de Grinberg y la colaboración de Merton. Asimismo, es posible establecer conexiones con otros actores y proyectos latinoamericanos, a partir de la presencia de Merton como nodo de las redes que manifiestan las revistas y la correspondencia.

Redes y archivos: intereses comunes y el involucramiento de Merton en la contracultura de los años ' 60

Hacia fines de los años 50, Thomas Merton experimentó una especie de epifanía que modificaría no solo su vida monástica en el claustro, sino sobre todo sus ideas acerca de su participación en las cuestiones sociales de la década de 1960. El momento epifánico ha sido descripto por el propio Merton en Conjectures of a Guilty Bystander (1968, p.153-154). Desde el momento en que Merton advierte que los muros de la abadía son una separación ilusoria, sin fundamentos reales, del resto de los seres humanos; y dado que la diferencia entre él como monje y los extraños no tiene sustancia, se produce un cambio en su relación con el mundo exterior al monasterio. Mark Meade estudia el intercambio epistolar de Merton con Victoria Ocampo como 
una temprana manifestación de la apertura o, cuanto menos, del aumento del involucramiento e interés de Merton por lo que pasaba extramuros (2013, p.171).

Para inicios de los años '60, las obras de Merton circulaban ya por Latinoamérica: en 1961 Sudamericana publicó el volumen 1 de las Obras Completas (fue el único tomo que llegaría a salir de prensa) y algunos de sus textos en prosa aparecían en revistas con cuyos directores Merton intercambiaba correspondencia (por ejemplo, el caso mencionado de Sur y Victoria Ocampo). Los escritos de Merton en revistas latinoamericanas tienen que ver, más allá de la temática específica de cada uno, con el ideario interamericanista de Merton. De hecho, en el caso de Sur, le escribe a Victoria Ocampo porque, sostiene, se siente identificado con el ideal promovido por Sur. ${ }^{1}$

Por la misma época en que Merton escribía a Ocampo y Sudamericana publicaba sus Obras Completas, un Miguel Grinberg edita el primer número de Eco Contemporáneo (noviembre-diciembre 1961). El volumen reúne un ensayo sobre la poesía brasileña, y artículos sobre los nadaístas en Colombia, el cine en Argentina, el neofascismo en Argentina, y los poemas Cuba 1960 de LeRoi Jones y America, de Ginsberg. Las temáticas abordadas en cada uno de estos textos ponen de relieve el interamericanismo por un lado, la adhesión a autores/programas contraculturales por otro, y además, el interés por distintos modos de expresión artística y sus connotaciones político-ideológicas. Ezequiel Gatto afirma: "Que la creación sea el eje de un imaginario político modifica profundamente aspectos esenciales de las relaciones entre aquellos que resisten creando" (Gatto, 2010, p.3).

A diferencia de la relación entre Merton y Sur, la otra revista argentina con la que mantuvo asiduo contacto y colaboración, Merton desconocía la existencia de Eco. A principios de los años 60 Grinberg era un joven ignoto, su emprendimiento editorial era manifiestamente contracultural, y ambas razones son causa suficiente de que el monje no supiera de la existencia de la revista (a diferencia de Sur y su directora Victoria Ocampo, de renombre internacional). De ahí que, mientras en la relación Merton-Ocampo fue el monje quien escribió la primera carta hacia Argentina, en el caso Merton-Grinberg es el joven quien se dirige al estadounidense primero. Le escribe el 5 de mayo de 1963, cuando la revista ya lleva dos años publicándose, y en un estilo sumamente informal, en papel membretado de "Eco Contemporáneo. Revista InterAmericana", se presenta:

Dear Merton, Here I am, Ernesto Cardenal gave me your address, I've mailed for you the 4 issues we have published, I know you're interested in Latin America [...] I'm 25, poet $[\ldots]$ my group is preparing literary revolt in Argentina [...] I want to print a Bulletin for the [Inter-American] Action [...] i[sic $]^{2}$ Are you interested? Please, answer soon... (Carta manuscrita, conservada en el Merton Center, Bellarmine University). ${ }^{3}$

\footnotetext{
${ }^{1}$ En la primera carta a Ocampo, Merton afirma: "El propósito principal de esta carta es simplemente hacerle saber mi admiración y decidido apoyo a Sur [...] le aseguro mi más completo acuerdo con todo lo que Sur representa [...]" (Merton; Ocampo: 2011, p.65).

${ }^{2}$ En inglés no se utiliza el signo de apertura de la interrogación. Es probable que Grinberg lo supiera, ya que su escritura, salvo por ese detalle, es en un correctísimo inglés; pero la trasferencia desde el castellano en la utilización del signo de puntuación se da por la rapidez de la escritura.

3 "Querido Merton: Acá estoy, Ernesto Cardenal me dio su dirección, le he mandado los 4 números que hemos publicado, sé que está interesado en Latinoamérica [...] Tengo 25 años, soy poeta [...] mi grupo está preparando la revuelta literaria en Argentina [...] quiero imprimir un Boletín para la Acción [Inter-americana] ¿Está interesado? Por favor responda pronto" (T.A.).
} 
En unas pocas líneas Grinberg se presenta, habla de su revista, de su "grupo", del movimiento Acción Interamericana y, a boca de jarro, le pregunta a Merton si está interesado en colaborar. La respuesta de Merton es también bastante descontracturada. El 21 de junio de 1963 le contesta:

I am very interested in your project, but I am a very bad correspondent, since I have a great deal to do and I hate to write letters without thinking. [...] The whole question of inter-American contacts and exchange is of the greatest importance [...] I am glad you are in contact with Ernesto [Cardenal]. [...] Do believe me in deep union and agreement with the forces of life and hope that are struggling for the renewal of the true cultural and spiritual vitality of the "new work" which is sometimes so tired, so old and so shabby [...] But the forces of life must win. ${ }^{4}$ (Merton, 1993, pp.196-197)

De esa respuesta se desprenden algunas líneas que muestran las coincidencias entre Merton y Grinberg: el inter-americanismo, las redes de las que ambos forman parte (con Merton como nodo central) y el deseo de un cambio que, por contraponerse al orden existente, podemos catalogar como contracultural. Junto con la carta, Merton envió varios textos que Grinberg promete en su respuesta incluir en el número 6. De hecho, el siguiente número de la revista fue sobre el 6/7 apareció hacia fines de 1963, e incluye la traducción al castellano de dos poemas, "Canción para ninguno" y "Y así, adiós a las ciudades." (Eco Contemporáneo 6/7, pp.92-93); y en la sección "Cartas", una de Merton (pp.169-170), junto con las del nicaragüense Pablo A. Cuadra, el estadounidense Lawrence Ferlinghetti, el indio Malay Roy Choudhury, el colombiano Gonzalo Arango y el argentino Julio Cortázar. En el mismo número se publican las respuestas de Merton a la "Encuesta americana. Arte y libertad" (que Grinberg le había enviado en carta mecanografiada el 18 de septiembre de 1963) ${ }^{5}$. La cantidad de textos escritos por Merton publicados en este número ponen en evidencia, por un lado, el entusiasmo del monje, que se reconocía ante todo escritor y enviaba varios manuscritos a sus corresponsales editores de revistas. Por otro lado, demuestran la admiración de Grinberg por Merton, y el prestigio que daba a su publicación la presencia de nombres reconocidos que se iban integrando a la hermandad inter-americana.

El 17 de enero de 1964, Grinberg envía en su carta una invitación a Merton: "Poets are meeting in Mexico City from the 5th to the 15th of february. It would be great to have you with us. It is going to be the FIRST AMERICAN ENCOUNTER of the NEW SOLIDARITY." (Carta mecanografiada conservada en el Merton Center). Y si bien Merton no puedo asistir al encuentro, envió el "Mensaje a los poetas", donde quedan plasmadas algunas cuestiones fundamentales en el ideario que comparten ambos sobre la poesía, la creación y la libertad, como se verá más adelante.

\footnotetext{
4 "Me interesa mucho tu proyecto, pero soy un mal corresponsal, porque tengo mucho que hacer y detesta escribir cartas sin pensar. [...] Todo el tema de los contactos e intercambios interamericanos es de suma importancia [...] Me place que estés en contacto con Ernesto [Cardenal] [...] Creeme que estoy en profunda unión y de acuerdo con las fuerzas de vida y esperanza que luchan para lograr la renovación de la verdadera vitalidad cultural y espiritual del "nuevo trabajo" que suele estar tan cansado, viejo y tan [...] pero las fuerzas de la vida deben triunfar" (T. A.).

${ }_{6}^{5}$ En existencia en el Merton Center de Bellarmine University.

6 "Los poetas nos vamos a reunir del 5 al 15 de febrero en Ciudad de México. Sería grandioso tenerte con nosotros. Será el PRIMER ENCUENTRO AMERICANO de la NUEVA SOLIDARIDAD” (T. A.).
} 


\section{Imaginarios compartidos}

Si bien los textos de Merton publicados en Eco no son muchos ${ }^{7}$, es posible estudiar el ideario compartido entre Merton y Grinberg a partir de esas páginas y del archivo epistolar mertoniano que se conserva íntegro en Bellarmine University (Kentucky, EEUU). Conviene revisar no solamente las cartas Merton-Grinberg, sino también otras de y a integrantes de las redes intelectuales que se fueron conformando, donde las referencias a Eco y a otros actores involucrados en publicaciones de la época permiten reconstruir los imaginarios e idearios compartidos.

El artículo editorial del número 6/7 de Eco, significativamente titulado "Nueva Solidaridad", señala esta progresiva ampliación del grupo: "Lo que en principio fue un eje entre México y Buenos Aires, entre El Corno Emplumado y nosotros, se convierte ahora en el prólogo de un Movimiento sin precedentes en la historia continental." (Eco Contemporáneo 6/7: 3) El artículo remite a los "hermanos" de otros países que respondieron al llamado, y enumera Venezuela, Paraguay, Uruguay, Ecuador, Perú, Brasil, Colombia, Usa [sic], Nicaragua, Puerto Rico, México y Canadá. "Días de alerta en las calles del continente, flores solares abriéndose en el momento inicial. Todo está por hacerse" (p.3). La conclusión del brevísimo texto tiene implicancias a la vez políticas y artísticas. El movimiento recién comienza, llama a la acción, y hay mucho por llevar a cabo. Estas ideas se encuentran en varios textos de Merton, quien compartiría, así, el imaginario (o parte del mismo) propugnado por Eco Contemporáneo. En ese imaginario compartido pueden distinguirse tres nociones: el inter-americanismo, la novedad (que no se propone como algo gratuito, sino por la necesidad de cambio), y el arte como medio para esa renovación.

\section{Inter-americanismo}

Como se dijo antes, el primer punto de contacto entre Merton y Grinberg, evidente en la carta que da inicio al intercambio, es el inter-americanismo, al que el monje da importancia vital. En otro trabajo ${ }^{8}$ hemos destacado la preeminencia de corresponsales latinoamericanos en el epistolario mertoniano: seis nicaragüenses, cuatro argentinos, dos chilenos, un venezolano, un cubano y un brasileño. (Raggio, 2018, p.26) Las cartas de Merton a estos latinoamericanos están compiladas en The Courage for Truth (Merton, 1993); y en todas ellas, además de cuestiones relativas a la creación literaria de cada uno, a los viajes que emprendían o los hechos de la vida personal, siempre Merton se interesa por la situación de los países del sur, por la posibilidad de aprender de los latinoamericanos, y por la necesidad de estrechar lazos entre las dos Américas.

Desde su primer número, correspondiente a noviembre-diciembre de 1976, Eco contemporáneo lleva el subtítulo "Revista Interamericana”, y la nota editorial se pregunta: “¿Por qué no todos los 'sub-americanos' unidos y enfrentando mutuamente las necesidades que solos y en conjunto podamos resolver?" (Eco: número 1, p.9). El interamericanismo de la revista es, en ese primer momento, una unión de los "sub-americanos"; Grinberg juega con los prefijos sub- y

${ }^{7}$ En el número 6/7 aparecen dos poemas y una carta; en el 8/9 el "Mensaje a los poetas" y en el número 13, en forma póstuma, "La lluvia y el rinoceronte".

${ }^{8}$ Marcela Raggio. Thomas Merton: el monje traductor. Buenos Aires, Corregidor: 2018. 
sud-, y contrapone lo que les es propio a las imitaciones de Europa y Estados Unidos, que quedan descartadas de plano.

En el número 2, correspondiente a enero-abril de 1962 el interamericanismo se desprende de ciertos elementos paratextuales: la publicidad de City Light Books, la mítica librería de Ferlinghetti en San Francisco; la nómina de ciudades en las que Eco tiene corresponsales (Río de Janeiro, Lima, La Paz, Quito, Cali, Nueva York), y tal como en el primer artículo editorial también aquí una pregunta: “¿América para quién?” (Eco, número 2, p.5).

En la página 112 del número 3, mayo-julio de 1962, una publicidad indica que las redes entre revistas se van expandiendo: El Corno Emplumado / The Plumed Horn aparece con la descripción "una revista de la Nueva Era abierta a todas las voces desde la ciudad de México". La revista, editada por Margaret Randall y quien era en ese entonces su esposo, Mondragón, es otra de las publicaciones latinoamericanas con las que también Merton colaboró. Los lazos con El Corno se hacen explícitos en la nota editorial del número 5: "Con la revista 'El Corno Emplumado' De México hemos concretado un eje, primer peldaño de la Integración, apertura de la Acción Interamericana" (Eco, número 5, 1963, p.4).

El inter-americanismo, que va dando forma a la revista y que se mantiene a lo largo de los trece números, aparece en la carta de Merton a Grinberg que éste publica en el número 6/7 (el que formalmente indica el inicio de la relación editorial entre ambos). En esa carta Merton sostiene: "...tengo muchísimo otros amigos en el resto del Hemisferio [Sur] en los que detecto una gran esperanza de despertar y de vida". (Eco 6/7, p.170) Entre los amigos que menciona en ese párrafo se cuenta Ernesto Cardenal, que es quien ha dado el dato de Merton a Grinberg, ya que el nicaragüense estaba en contacto con el argentino y había publicado en el número 5 de Eco. Tal como se advierte en los distintos números de Eco, también los muchísimos amigos de Merton provienen de diversos ámbitos e ideologías, y se reúnen en emprendimientos como esta revista buscando algo nuevo.

\section{La novedad como necesidad de cambio}

Carlos Gradín propone que Eco Contemporáneo fue una de las publicaciones que buscaban poner nombre a la necesidad de cambios en los nuevos tiempos: "Eco Contemporáneo, precisamente, fue la revista que Miguel Grinberg fundó junto a un grupo de amigos en diciembre de 1961, en Buenos Aires, y en la que puede leerse otra poética de los nuevos tiempos, y el afán por darles un nombre e imaginar su evolución" (2010, en línea). La propia revista sufre, a lo largo de sus trece números, una evolución que indica que está signada por la necesidad de cambio: no se atiene a un perfil determinado, ni a un enfoque ni ideología concreta. De los números iniciales donde predomina la poesía, Eco muestra luego interés por el cine, la danza., las artes plásticas, la ecología, la política etc. En todos los casos, se busca incorporar voces nuevas, las que han surgido o que buscan responder a las necesidades de la nueva década. Continúa Gradín: "Eco va a hablar de una búsqueda de nuevos modos de pensar el mundo, el arte y las relaciones personales, en una utopía de ampliación de la consciencia y las capacidades creativas" (Gradín, 2010).

"Nos gestaremos a nosotros mismos y creceremos sin prisa ni miedos" (Eco número 2, p.5). La aseveración señala la ruptura con todo lo precedente, la negación de filiaciones, y por lo tanto, el deseo de cambio y novedad. Esto se ve acentuado en el editorial del número 4 
(diciembre 1962), que comienza exclamando "Dijimos A OTRA COSA'. Esto implica dar vuelta la hoja, no como actitud circunstancial sino como gesto definitivo, a partir del cual todo queda por hacer. [...] Resolvemos edificar un nuevo modo de vida con visión de futuro" (p.1). Thomas Merton responde a ese llamado a crear para cambiar, y en la primera carta que responde a Grinberg (publicada en el número 6/7 de la revista), afirma: “[...] sépanme en profunda unión y de acuerdo con las fuerzas vitales y esperanzadas que luchan por la renovación y una verdadera vitalidad espiritual y cultural para este 'nuevo mundo' 10 a menudo tan fatigado, tan viejo y tan vil" (p.170). Para el Merton de la década de 1960, las revistas literarias y culturales son un medio válido, no solamente para establecer contacto con Latinoamérica, sino para renovar el mundo, algo que suena ambicioso y general, a menos que se acote al campo de las expresiones artísticas.

\section{El arte como medio para la renovación}

Gatto (2012) enumera la serie de prácticas artísticas por las que se interesó Eco:

[...] es posible comprenderla como un espacio atravesado medularmente por prácticas artísticas (especialmente la literatura, pero también el cine, el teatro, la plástica, la danza y la música) vinculadas de diversas maneras a experiencias políticas o militantes emancipatorias alejadas de las líneas quizá más hegemónicas de la política del período. (pp.173-174)

Si bien es posible distinguir una suerte de evolución o cambio en los intereses de la revista a medida que pasa el tiempo, en los primeros números (los que envía a Merton con la primera carta) es bien evidente la preponderancia de poemas, notas sobre cine, artes plásticas y danza.

En esa comunicación mediante el arte, Merton encuentra los ecos de su propio proyecto. Y si bien es cierto que Merton es un intelectual comprometido, más allá de las limitaciones propias de su vida monástica, en carta a Grinberg, fechada el 12 de julio de 1964, le recomienda: "There has to be more poetry. For exploration. The exploration by poetry is the kind most needed now. Drama too, art, music, dancing, seminars, silence" 11 (carta manuscrita conservada en el Merton Center). La enumeración parece un repaso de los temas abordados en Eco. De hecho, Merton se está refiriendo a las actividades de exploración que Grinberg proponía en su carta anterior, del 7 de julio, desde Washington D.C. (copia disponible en el Merton Center), a través del teatro y de seminarios de especialistas en diversos temas. La carta, posterior al encuentro entre Merton y Grinberg en Getsemaní tres meses antes, probablemente sea una continuación de las espaciadas charlas que mantuvieron durante la estancia de Grinberg en la abadía. En Memoria de los ritos paralelos (2014) Grinberg describe esa visita marcada por silenciosos paseos por los bosques y jardines de Getsemaní (p.22). En el número 6/6 de Eco, reclama ese mismo silencio como cualidad necesaria para la creación, que va a producir algo nuevo: "Días de alerta en las calles del Continente, flores abriéndose en el momento inicial. Todo está por hacerse" (Eco 6/7, p.3).

\footnotetext{
${ }^{9}$ Mayúsculas en el original.

${ }^{10}$ En negrita en el original.

11 “Tiene que haber más poesía. Para la exploración [en castellano en el original]. La exploración a través de la poesía es la que más se necesita hoy. También teatro, arte, música, danza, seminarios, silencio.” (T. A.)
} 
La referencia al momento inicial está presente también en el texto que Merton escribió para el Encuentro del Movimiento Nueva Solidaridad que se llevó a cabo en México en 1964, y que se reproduce en el número 8/9 de Eco. En las líneas iniciales del "Mensaje" Merton exclama:

Los que somos poetas sabemos que el motivo de un poema no es descubierto hasta que ese poema existe. [...] Esta reunión es una explosión espontánea de esperanzas. [...] Es expresión viva de la creencia de que hay ahora en nuestro mundo gente nueva, nuevos poetas que no están bajo la tutela de sistemas políticos establecidos ni de estructuras culturales -sean comunistas o capitalistas- pero que se atreven a tener esperanza en su visión de la realidad y del futuro. (p.60)

La reproducción del "Mensaje" en el número correspondiente al invierno de 1965, un volumen que en la ficha editorial se señala pertenece a la "era ulterior", demuestra que Eco está constantemente reinventándose, en un permanente momento inicial. Y si Merton había escrito esas palabras a finales de 1963, casi dos años después Grinberg y sus coetáneos siguen encontrando los mismos ecos de novedad y de centralidad de la poesía como acto creador.

\section{Canon y archivo: redes en Eco contemporáneo, cartas y diarios}

Valeria Manzano sostiene: “La búsqueda de un 'nosotros' fue distintiva de la actividad cultural, muchas veces febril, de Grinberg. Ya desde el primer número de Eco Contemporáneo los esfuerzos se centraron en incluir voces que se reconocían similares en las Américas" (p.120). Como se ha ido señalando a lo largo de las páginas previas, tanto en las cartas como en las publicidades de otras revistas, anunciadas en los diferentes números de Eco, y en la concreción del Encuentro de poetas en México, es posible advertir las redes que se van conformando entre americanos del norte y del sur, y entre Eco y varias publicaciones del continente. Particularmente nos interesa aquí realizar un cruce de datos que surgen de Eco y de las cartas Merton-Grinberg, sumadas a exploraciones en otros archivos de los escritores implicados. En todos los casos, Merton es el nodo central, ya que para la década del 60 era una figura reconocida entre los poetas, los pacifistas, los interamericanistas y diversas "tribus" culturales, literarias y religiosas.

Como se vio antes, Grinberg escribe por primera vez a Merton porque Ernesto Cardenal le ha dado la dirección; y cuenta que ha fundado Acción Interamericana, un movimiento del que Henry Miller ha aceptado ser presidente honorario. En su respuesta, Merton le dice: "I am glad you are in contact with Ernesto" 12 . Y agrega: "Henry Miller is a good friend of mine and I think he has very good insight into this problem. He has said many really urgent things about the question of our modern world and where it is going"13 (Merton 1993, p.196). En el diario de Merton, en la entrada correspondiente al 11 de agosto de 1962, se lee: "Henry Miller's tremendous essay on Raimu [en The Wisdom of the Heart] deeply significant, touches the real nerve of our time [...]" ${ }^{14}$ (Merton 1996, p.237). Por la misma época, Merton le escribe a

\footnotetext{
12 "Me alegra que estés en contacto con Ernesto." (T. A.)

13 "Henry Miller es buen amigo mío y creo que comprende bastante bien el problema. Ha dicho varias cosas urgentes sobre el tema de nuestro mundo moderno y hacia dónde se dirige".

14 "El tremendo ensayo de Henry Miller sobre Raimu profundamente significativo, toca el verdadero nervio de nuestro tiempo" (T. A.)
} 
Cardenal: "He estado en contacto con Henry Miller. Ha escrito recientemente algunos ensayos extraordinarios, especialmente un libro titulado La sabiduría del corazón [...]" (Merton; Cardenal 2003, p.98).

Al ser amigo común, Miller representa un nexo entre Merton y Grinberg, una lectura de ambos, y a la vez Merton, en su papel de maestro del joven argentino, también escribe a Miller recomendándoselo: "By now you must have seen Grinberg himlsef in person. He is a promising young guy and the thing I like best about him is that he is free of the bitterness and frustration and self-pity that is eating up so many of the young poets"15 (Merton 1993, p.280). De hecho, Grinberg visitó a Miller en septiembre de 1964, y sus Memorias de los ritos paralelos se cierran significativamente con la traducción de la carta de Merton a Miller, y una foto del joven Grinberg y Miller en California.

Palabras similares a las de la carta a Miller son las que usa el monje para encomiar a Cardenal cuando le escribe a Grinberg en la primera carta que le alegra saber que Cardenal y él se conocen: "He [Cardenal] will be doing great work, and is already at the height if his powers, perhaps, with great promise of more development. He will be one of the most significant voices in the two Americas. I am warmly attached to my friends in Nicaragua who are writing fine poetry [...]"16. A la vez, en este juego de cartas que se entrecruzan, dialogan, reverberan, Merton escribe a Cardenal acerca de Grinberg el 10 de marzo de 1964:

Miguel Grinberg está aquí, y estoy muy contento de haberlo conocido. Su encuentro en México [el encuentro del Movimiento Nueva Solidaridad] parece estupendo, ciertamente el Espíritu se está moviendo a través de Sudamérica y Latinoamérica en general, y este movimiento de poetas y artistas hacia una nueva conciencia espiritual es lo más esperanzador que he visto en el mundo últimamente. (Merton; Cardenal, pp.133-134)

Ese esperanzador movimiento $-\mathrm{o}$ al menos, las redes que lo van produciendo- se advierten en el número 6/7 de Eco donde, como se dijo antes, aparecen por primera vez los escritos de Merton. En la lista de miembros de la redacción de la revista, Henry Miller aparece como "Sacerdote honorario" de la Acción Poética Interamericana. El volumen incluye un análisis de Rafel Squirru sobre la correspondencia Miller - Lawrence Durrell, los ya mencionados textos de Merton, y el poema "Nindirí", de E. Cardenal. Hay además una de las entregas de Grinberg (la tercera) sobre "Mufa y revolución"; y este número doble de la revista coincide con el lanzamiento oficial, anunciado en las páginas 3 y 4, de la Acción Poética Interamericana. Al llamado, dice Grinberg, han respondido poetas del norte y del sur. Las cartas transcriptas en las páginas finales, el lanzamiento conjunto de la Casa de las Américas en México (mencionada también en la página 3), las publicidades de revistas como la nicaragüense El pez y la serpiente (otra revista con la que colaboró Merton) y la italiana The New Morality. Revista de arte y literatura, plasman un ambiente de creación, esperanza e intercomunicación, vínculos que viajan a través del correo y de las visitas de estos poetas, intelectuales y activistas que quieren hacer una revolución, aunque no coincidan en todos los puntos.

15 "Ya debes haber visto a Grinberg en persona. Es un joven prometedor y lo que más me gusta de él es que está libre de la amargura y frustración y autocompasión que devora a la mayoría de los buenos poetas jóvenes" (T. A.).

16 “[Cardenal] está haciendo un trabajo magnífico, y ya se encuentra en la cúspide de sus poderes, tal vez, con la promesa de más desarrollo. Será una de las voces espirituales más importantes de las dos Américas. Estoy cálidamente vinculado a mis amigos de Nicaragua que están escribiendo buena poesía [...]” (T. A.). 


\section{Conclusiones: "intercambio de vital importancia"}

En trabajos previos hemos estudiado el valor que Thomas Merton otorgaba al diálogo e involucramiento en la cuestión latinoamericana. ${ }^{17}$ En sus cartas a escritores de Sudamérica manifiesta claramente su interamericanismo, que no se limita a una declamación vacía, sino que lleva a la práctica compartiendo libremente sus escritos con varias revistas (Sur, El Corono Emplumado, El pez y la serpiente, Eco Contemporáneo entre otras). En el caso de Eco, si bien los textos de Merton no abundan, es posible señalar por un lado la confluencia de pensamientos con Grinberg en lo relativo a la necesidad de revolucionar el mundo, aunque la revolución no sea armada sino, como firma en la despedida de una de sus cartas, "Up with the revolution of tulips. [...] Love and Peace, silence, movement of planets"18 (Merton 1993, p.200). Por otro lado, las cartas ponen de relieve también los movimientos si no de planetas, al menos de jóvenes poetas americanos que escriben, se trasladan, se leen y se reúnen en un gran encuentro a escala continental.

Desde su primera carta a Grinberg, Merton afirma que el interamericanismo implica in intercambio vital. Gran parte de sus escritos de la década de 1960 apuntan a construir ese interamericanismo e, incluso, a indicar la superioridad de Latinoamérica sobre el Norte, como en la paradigmática "Carta a Pablo A. Cuadra, acerca de los gigantes" (Sur 275: marzo-abril 1962). Las cartas Merton-Grinberg, y las líneas que de ellas y de Eco se desprenden para incluir a Henry Miller, a Ernesto Cardenal (mencionados en este artículo) y a tantos otros como el propio Pablo A. Cuadra, Margaret Randall, Sergio Mondragón, Allen Ginsberg, Lawrence Ferlighetti et al., manifiestan la importancia central que tuvo la relación entre las dos Américas, el papel que las publicaciones periódicas cumplieron en los diálogos e intercambios de los años ' 60 , y el valor que el archivo reviste para abordar el estudio de los proyectos culturales e ideológicos de los revolucionarios que no se dejaron llevar por las propuestas de las potencias enfrentadas en la época. Como escribe Grinberg, "Rechazamos por vanas las jerarquías y categorías fundadas en torno de lo que se da por llamar izquierda o derecha, con todas su mitologías e historias colaterales. El pensamiento y la inteligencia son inclasificables. La realidad, una sola: querer que esta siga así, o que cambie" (Eco 4, p.2). El cambio como motor de la realidad y de la revista no puede sino conseguir el apoyo de Merton, el inclasificable monje cisterciense que buscaba, por la época en que Grinberg le escribió, llegar a los acontecimientos del mundo más allá del claustro y generar lazos fraternos sobreponiéndose a los límites nacionales, generacionales, religiosos y lingüísticos. Eco fue una plataforma para ese proyecto intercontinental, poético y cultural.

\footnotetext{
${ }^{17}$ Pueden verse nuestros trabajos "Thomas Merton's Americanism: a study of his ideas on America in his letters to writers" (Revista de Estudios Norteamericanos 20: Universidad de Sevilla, 2016), "Las ideas de Thomas Merton en Latinoamérica: publicaciones de Merton en Sur" (Prismas Revista de Historia Intelectual 23: Universidad Nacional de Quilmes, 2019) y Thomas Merton, traductor de poesía latinoamericana (Buenos Aires: Corregidor, 2018).

18 "Viva la revolución de los tulipanes. [...] Amor y Paz, silencio, movimiento de planetas” (T. A.).
} 


\section{Referencias Bibliográficas}

Gatto, E. (2012). "El nuestro es un combate de creación: la revista Eco Contemporáneo, Argentina 1960-1969." CS No. 9, 169-198, enero-junio 2012. Cali, Colombia.

Gradín, C. (2010) "Para una genealogía de la mufa. Miguel Grinberg y la revista Eco Contemporáneo (1961-1969)". El interpretador número 36. marzo 2010. Disponible en línea: https://revistaelinterpretador.wordpress.com/2017/03/16/para-una-genealogia-dela-mufa-miguel-grinberg-y-la-revista-eco-contemporaneo-1961-1969/.

Grinberg, M. (2014). Memoria de los ritos paralelos. Buenos Aires: Caja Negra.

Manzano, Valeria. (2017). "Fraternalmente americanos: el Movimiento Nueva Solidaridad y la emergencia de una contracultura en la década de 1960". Iberoamericana, XVII, 66 (2017), 115-138.

Meade, M. (2011). "From Downtown Louisville to Buenos Aires: Victoria Ocampo as Thomas Merton's Overlooked Bridge to Latin America and the World”, Merton Annual, ${ }^{\circ} 26$.

Merton, M. (1968). Conjectures of a Guilty Bystander, Nueva York: Doubleday . (1993). The Courage for Truth. Letters to Writers. Ed Christine Bochen. Nueva York: Farrar, Strauss, Giroux

Merton, T.; Cardenal, E. (2003). Correspondencia (1959-1969). Madrid: Trotta

Merton, T.; Ocampo, V. Fragmentos de un regalo. Correspondencia y artículos y reseñas en Sur. Buenos Aires: Sur 